\title{
Evaluating the functioning and impacts of funding schemes. New methodological developments of indicators
}

\author{
Benedetto Lepori ${ }^{1, *}$, Emanuela Reale $^{2}$ and Maria Nedeva ${ }^{3}$ \\ ${ }^{I}$ Centre for Organizational Research, University of Lugano, Switzerland \\ ${ }^{2}$ CERIS CNR, Italy and \\ ${ }^{3}$ Manchester Institute of Innovation Research, University of Manchester, UK \\ *Corresponding author. Email: blepori@usi.ch
}

\section{Introduction}

This special issue collects five articles that have been presented at the Science and Technology Indicators Conference organized by the European Network of Indicators Designers (ENID) in September 2011 in Rome. As such, they represent the final outcome of a selection process which started with about 100 abstract submissions for the conference itself and went then through, first, the selection process for the conference and, second, through an open call for articles for this special issue, and an external peer review process, to which 13 articles have been submitted. We gratefully acknowledge the many referees who kindly contributed - often at short notice - their insightful, critical, and constructive inputs to help us and the authors to produce the high-quality articles presented in this issue.

Expectedly from the organization of this process, these articles cover quite different topics in the field of design of indicators ranging from internazionalization of funding agencies (Reale, Inzelt, Lepori \& van den Besselaar, this issue), to the characterization of funding agencies selection processes (Thomas \& Nedeva; Neufeld \& Hornbostel, this issue), to the analysis of publication outputs in social sciences and humanities (Ossenblok, Engels \& Sivertsen, this issue) and, finally, to the investigation of relational structures of higher education systems in a regional context (Seeber, Lepori, Agasisti, Tijssen, Montanari \& Catalano, this issue).

Despite this topical diversity, we highlight two crosscutting topics which are common to some of these articles and which have been central more in general to the STI conference 2011. These are the use of (different types of) indicators in order to assess the organization and working of research funding systems on the one side, the recognition that research and higher education systems are characterized by a dense web of social relationships with deep implications for the system's structure, competition for resources and individual performance (Stokman 2011) on the other side.

\section{The papers in a nutshell}

The article of Reale et al. deals with the construction of indicators to measure the internationalization of funding agencies, by reporting the results of a forum promoted by the European Science Foundation with the participation of different national agencies. In the tradition of sociology of indicators, it frames it as a process of social debate and construction of consensus between the involved agencies (Barré 2001; Lepori, Barré and Filliatreau 2008), where joint decisions have been taken concerning the objective of the exercise, the framework for the construction of indicators and the selection of those to be included in the final list. The article displays, first, the importance of a thorough understanding of the functions of funding agencies in the research policy system (Braun 1998), as well of overall patterns of internationalization (Edler and Flanagan 2011) in order to design a coherent indicator systems and, second, that dialogue and participation are critical to develop indicators which suit user's needs are accepted by them. 
The two following articles deal with different aspects of the funding agencies selection process of grant applications. Thomas and Nedeva develop a systematic framework to characterize applicants to the European Research Council Starting Grants, covering those key features which are expected to impact on their ability to pursue frontier research. Further, they provide a preliminary testing based on a sample of applicants to that scheme. While the use of a larger sample, which allows for statistical analysis, as well as longitudinal studies will be required to fully assess the usability of the scheme, their work demonstrates how from extant research it is possible to develop and operationalize a characterization framework tailored to the specific program goals and taking into account the balance between different objectives (rather to adhere to a generic definition of what research quality is).

Neufeld and Hornbostel deal with a rarely investigated characteristic of funding schemes, namely their ability to promote self-selection of applicants based on the stated selection rules. This issue is both of scholarly and practical relevance: for the first, analyzes of selection processes based on effective applicants are likely to oversee a central component of the process itself, namely that a number of potentially eligible applicants might not apply because they perceive their success chances as too low. For the second, from the perspective of funding agencies, self-selection is a desirable outcome as it reduces the workload in evaluation, but only at the condition that non-applicants are those not matching the wished profile for that scheme. The empirical results presented show that self-selection works well, in the sense that most eligible applicants not matching the profile for the scheme considered do not apply, namely those with a publication record well below the recommended standards. On the one side, the low performers considered managed developing a successful research career and, on the other side, the selection process was not really able to discriminate between good and excellent scientists. This suggests that there are limitations to the ability of identifying at an early stage of career the most promising researchers and, accordingly, that a plurality of funding schemes should be maintained to support the variety of career paths observed.

The article by Ossenblok, Engels, and Sivertsen deals with a broader issue related to the impact of funding systems. Namely, by using national publication databases in Norway and Flanders, they investigate for social sciences and humanities (SSH) how funding schemes impact on publication behavior of researchers. While both countries have introduced performance-based funding, there are differences in the reference set of publications: the Flanders scheme included only publications indexed in the Web of Science (WoS), while the Norwegian scheme included all items in the national publication database - providing however a differentiation in two quality levels. The data show a similar increase in both countries for the share of publications in English, but diverging evolutions concerning the share of publication output indexed in WoS, which increased in Flanders and remained stable in Norway; in the latter country, there has been a strong increase in the share of top-level publications in the national classification scheme. These results confirm on a large scale previous evidence that incentive schemes influence publication behavior; further, they show the value of analysis based on national publication databases to characterize publication behavior of scientists, especially in the case of SSH.

Finally, the article by Seeber et al. deals with the analysis of competitive and cooperative relationships between higher education institutions in a regional context, the Lombardy region in Italy. Following developments in social network analysis and economic sociology, the article argues that, to analyze the functioning of higher education, it is critical, first, to provide indicators characterizing relationships between individual organizations and, second, that it is important to focus on the interactions between the research and the teaching arena as well. Following this insight, they develop a rich set of indicators and empirical tests on research collaborations on the one side and competition for students on the other side. From their data, they demonstrate that, as an outcome of geographic centrality, universities located in the Milan metropolitan area are able to attract students from the whole region; in the context of a system characterized by a funding allocation which is broadly proportional of students, these universities concentrate a large share of resources and become central in the research arena as well. The authors finally speculate that this mechanism reduces international competitiveness of Italian universities, as they need to train large number of students to acquire resources for research.

\section{References}

Barré, R. (2001) 'Sense and Nonsense of S\&T Productivity Indicators. The Contribution of European Socio-Economic Research to the Benchmarking of RTD Policies in Europe', Proceedings of a Conference Organized by the European Commission Directorate General for Research, Brussels, March 15-16, 2001.

Braun, D. (1998) 'The Role of Funding Agencies in the Cognitive Development of Science', Research Policy, 27: 807-21.

Edler, J. and Flanagan, K. (2011) 'Indicator Needs of Research Internationalisation Policy', Research Evaluation, 20/1: 7-17.

Lepori, B., Barré, R. and Filliatreau, G. (2008) 'New Perspectives and Challenges for the Design and Production of S\&T Indicators', Research Evaluation, 17/1: 33-44.

Stokman, F. (2011) The Crucial Role of Cooperation and Competition in Social Networks for Science and Technology Indicators, Paper presented at the ENID S\&T Conference, Rome, September 2011. 